\title{
Chondrocyte clocks make cartilage time-sensitive material
}

\author{
Karen M. Doody and Nunzio Bottini \\ La Jolla Institute for Allergy and Immunology, La jolla, California, USA.
}

\begin{abstract}
Circadian rhythms mediated by both central and tissue-specific peripheral clocks allow for the synchronization of biological processes with diurnal cycles such as activity and rest. Disruption of these rhythms can be caused by altered sleep-awake patterns or by pathological conditions and can initiate or exacerbate human disease through mechanisms that are only partially understood. In this issue, Dudek et al. identify a chondrocyteautonomous cartilage clock and demonstrate that expression of an important circadian pacemaker, BMAL1, decreases during osteoarthritis progression. They show that chondrocyte-specific deletion of BMAL1 leads to cartilage degradation and disruption of key pathways, shifting cartilage homeostasis toward a catabolic state. These findings provide insight into the interplay between circadian rhythm and cartilage in osteoarthritis.
\end{abstract}

In order to isolate the role of BMAL1 within the cartilage clock, the authors used BMAL1-floxed mice crossed with a chondrocyte-specific Col2a1-Cre deleter strain. It was previously demonstrated by Takarada et al. that global deletion of BMAL1 results in decreased body size and weight and defective chondrogenesis, locomotor ability, and metabolism (11). The same group had also crossed BMAL1-floxed mice with a chondrocyte-specific Col2a1-Cre deleter strain (12) and showed that chondrocyte-specific deletion of BMAL1 results in bone development defects similar to those seen in global KO mice (11). The Dudek et al. study (9) complements the previous study by addressing the role of BMAL1 in cartilage homeostasis in adult tissues. Their study of adult cartilage homeostasis in cartilage-specific BMAL1-KO mice was facilitated by a lack of the defects in body weight and bone growth that were observed in the study by Takarada et al. This apparent discrepancy might be due to the fact that the two groups used Col2a1-Cre transgenic deleter strains that differ in the Col2a1 promoter-targeting strategy.

The authors first assessed the effect of BMAL1 deficiency on the function of central and peripheral clocks using a luciferase reporter assay to monitor the periodicity of PER expression in various tissues. They confirmed that Col2a1 Bmal1-KO mice have decreased circadian rhythms in hip cartilage, while PER expression cycles were normal in the suprachiasmatic nuclei (the central clock) and other tissues such as the lung and heart. Activity cycles were unaltered in Col2a1 Bmal1-KO mice, which also confirmed an intact central circadian clock and ruled out biasing of the study by central clock-dependent alterations of physical activity that would affect cartilage turnover.

\section{Time-sensitive nature of} cartilage

In addition to showing that cartilage contains its own clock, Dudek et al. showed that disrupting the clock by cartilage-specific dele- 
tion of BMAL1 resulted in progressive cartilage damage, specifically in the knees (9). Lesions appeared in the absence of changes in surrounding synovium and subchondral bone, pointing to chondrocyte-specific pathology. While the phenotype induced by cartilage-specific BMAL1 deficiency did not fully mimic human osteoarthritis, it does support the idea that disruption of the chondrocyte-intrinsic circadian rhythm can result in cartilage pathology. It should be noted that complete deletion of BMAL1 was not reported to result in defects in the cartilage matrix; however, $\mathrm{Bmal1}^{-/-}$mice have defective bone growth and locomotor defects that would alter mechanical input that could influence peripheral clocks.

\section{What makes the cartilage clock tick?}

The observations that cartilage BMAL1 expression decreases with age in mice and in patients with osteoarthritis and that deletion of BMAL1 in chondrocytes alone causes cartilage damage suggest that disruption of the circadian rhythm may predispose individuals to cartilage pathology. In order to better understand the underlying mechanisms connecting circadian rhythms to cartilage destruction, Dudek et al. performed a series of time-course RNA-sequencing experiments using hip cartilage to follow transcription pathways in Col2a1 Bmal1-KO mice compared with those in control mice over 24-hour periods. A large number of BMAL1-dependent genes were identified, the majority of which followed 24-hour cycles of expression and then lost their periodicity upon BMAL1 deficiency. Many of the genes studied were regulated in the night or early morning, showing a correlation with the activity cycles of mice. The authors then performed pathway analyses to identify key pathways controlled by BMAL1 in chondrocytes. One of the pathways identified was the TGF- $\beta$ pathway, which in Col2a1 Bmal1KO mice was skewed toward a catabolic pattern of signaling, similar to that observed in aging and arthritic human cartilage (13). A second pathway was the NFATC2 pathway, which was repressed in Col2a1 Bmal1-KO mice. NFATC2 is an important regulator of cartilage homeostasis, and its deletion, combined with cartilage-specific ablation of NFATC1 in mice, results in osteoarthritislike cartilage damage (14). Dudek et al. demonstrated that NFATC2 signaling was decreased in cartilage of Col2a1 Bmal1-KO mice, which correlated with decreased expression of anabolic factors such as collagen and aggrecan (9). Finally, the authors showed that NFATC2 expression in cartilage was controlled by CLOCK, a transcription factor and binding partner of BMAL1, and followed temporal expression patterns. These results constitute an initial elucidation of the molecular mechanism through which the peripheral clock influences cartilage homeostasis.

\section{Conclusions and future questions}

In this issue, Dudek et al. (9) demonstrate that cartilage contains a cell-autonomous circadian clock and that disruption of this clock results in a shift toward a chondrocyte catabolic state. In diseases such as osteoarthritis, it is well known that symptoms such as pain and stiffness have a time-of-day pattern, and diurnal patterns of activity and rest also may affect cartilage through variations in mechanical load and movement. The current study gives a first glimpse into the possible contribution of the cartilage-autonomous circadian clock to cartilage homeostasis and prevention of joint pathology. These findings suggest that further studies of the cartilage clock and its role in arthritis are warranted and raise several interesting questions. First, it will be interesting to know what causes BMAL1 expression to decrease during osteoarthritis progression and aging. Second, in this study, circadian gene expression networks were assessed in hip articular cartilage, while cartilage pathology in Col2a1 Bmal1-KO mice was only observed in knees. It will be important to understand whether cartilage clock regulation varies among different joints, as such a difference might theoretically contribute to the joint-specific manifestations of various forms of arthritis (15). Finally, and closely related to the site specificity of cartilage clocks, it is unknown how movement and mechanical load affect, and are affected by, the cartilage clock. Further investigation into the cartilage circadian clock would be of high relevance to rheumatology by improving our understanding of the etiology of osteoarthritis and potentially other arthritic entities, which might enable novel strategies for the prevention and treatment of these common conditions.

\section{Acknowledgments}

Rheumatology research in the N. Bottini laboratory is supported by NIH grants AR066053 and AI070544.

Address correspondence to: Nunzio Bottini, La Jolla Institute for Allergy and Immunology, 9420 Athena Circle, La Jolla, California 92037, USA. Phone: 858.752.6815; E-mail:nunzio@lji.org.

1. Reddy AB, O’Neill JS. Healthy clocks, healthy body, healthy mind. Trends Cell Biol. 2010;20(1):36-44.

2. Hansen J, Stevens RG. Case-control study of shift-work and breast cancer risk in Danish nurses: impact of shift systems. Eur J Cancer. 2012;48(11):1722-1729.

3. Marquie JC, Tucker P, Folkard S, Gentil C, Ansiau D. Chronic effects of shift work on cognition: findings from the VISAT longitudinal study. Occup Environ Med. 2015;72(4):258-264.

4. Parsons MJ, et al. Social jetlag, obesity and metabolic disorder: investigation in a cohort study. Int JObes (Lond). 2015;39(5):842-848.

5. Bellamy N, Sothern RB, Campbell J, Buchanan WW. Rhythmic variations in pain, stiffness, and manual dexterity in hand osteoarthritis. Ann Rheum Dis. 2002;61(12):1075-1080.

6. Bellamy N, Sothern RB, Campbell J. Rhythmic variations in pain perception in osteoarthritis of the knee. J Rheumatol. 1990;17(3):364-372.

7. Foldes K, Balint P, Gaal M, Buchanan WW, Balint GP. Nocturnal pain correlates with effusions in diseased hips. J Rheumatol. 1992;19(11):1756-1758.

8. Gossan N, Boot-Handford R, Meng QJ. Ageing and osteoarthritis: a circadian rhythm connection. Biogerontology. 2015;16(2):209-219.

9. Dudek M, et al. The chondrocyte clock gene Bmal1 controls cartilage homeostasis and integrity. J Clin Invest. 2016;126(1):365-376.

10. Gossan N, et al. The circadian clock in murine chondrocytes regulates genes controlling key aspects of cartilage homeostasis. Arthritis Rheum. 2013;65(9):2334-2345.

11. Takarada T, et al. Clock genes influence gene expression in growth plate and endochondral ossification in mice. J Biol Chem. 2012;287(43):36081-36095.

12. Terpstra L, et al. Reduced chondrocyte proliferation and chondrodysplasia in mice lacking the integrin-linked kinase in chondrocytes. J Cell Biol. 2003;162(1):139-148.

13. Blaney Davidson EN, et al. Increase in ALK1/ ALK5 ratio as a cause for elevated MMP-13 expression in osteoarthritis in humans and mice. Jimmunol. 2009;182(12):7937-7945.

14. Greenblatt MB, et al. NFATc1 and NFATc2 repress spontaneous osteoarthritis. Proc Natl Acad Sci U S A. 2013;110(49):19914-19919.

15. Pap T, Korb-Pap A. Cartilage damage in osteoarthritis and rheumatoid arthritistwo unequal siblings. Nat Rev Rheumatol. 2015;11(10):606-615. 